\title{
Article
}

\section{Mechanical characterisation of the lateral collateral ligament complex of the ankle at realistic sprain-Ilke strain rates}

Rochelle, David Christopher, Herbert, Anthony, Ktistakis, loannis, Redmond, Anthony Charles, Chapman, Graham and Brockett, Claire Louise

Available at http://clok.uclan.ac.uk/30326/

Rochelle, David Christopher, Herbert, Anthony, Ktistakis, Ioannis, Redmond, Anthony Charles, Chapman, Graham ORCID: 0000-0003-3983-6641 and Brockett, Claire Louise (2020) Mechanical characterisation of the lateral collateral ligament complex of the ankle at realistic sprain-like strain rates. Journal of the Mechanical Behavior of Biomedical Materials, 102 . p. 103473. ISSN 1751-6161

It is advisable to refer to the publisher's version if you intend to cite from the work. http://dx.doi.org/10.1016/j.jmbbm.2019.103473

For more information about UCLan's research in this area go to http://www.uclan.ac.uk/researchgroups/ and search for <name of research Group>.

For information about Research generally at UCLan please go to http://www.uclan.ac.uk/research/

All outputs in CLoK are protected by Intellectual Property Rights law, including Copyright law. Copyright, IPR and Moral Rights for the works on this site are retained by the individual authors and/or other copyright owners. Terms and conditions for use of this material are defined in the policies page. 


\section{Mechanical characterisation of the lateral collateral ligament complex of the ankle at realistic sprain-like strain}

rates

1 Abstract

2

\section{BACKGROUND}

3 Synthetic interventions continue to evolve with the progression made in materials

4 science, surgical technologies and surgical methods. To facilitate the evolution of

5 synthetic devices for lateral ankle repair a better understanding of the mechanical

6 properties and failure mechanisms of the lateral collateral ligament (LCL) complex

7 is required. This study aimed to improve understanding of the mechanical properties and failure modes of the LCL complex at strain rates representative of sprain.

\section{METHOD}

The LCLs were dissected from six human cadavers to produce individual boneligament-bone specimens. A mechanical testing device uni-axially loaded the ligaments in tension. Initially, preconditioning between two Newtons and a load value corresponding to $3.5 \%$ strain was conducted for 15 cycles, before extension to failure at strain rate of $100 \% . \mathrm{s}^{-1}$. The results were stratified by age, weight and body mass index (BMI) to explore potential correlations with ligament ultimate failure load or ligament stiffness.

RESULTS 
The mean ultimate failure loads and the $95 \%$ confidence intervals for the ATFL, calcaneofibular (CFL) and posterior talofibular (PTFL) ligaments were $263.6 \pm 164.3$

$\mathrm{N}, 367.8 \pm 79.8 \mathrm{~N}$ and $351.4 \pm 110.8 \mathrm{~N}$, respectively. A strong positive Pearson correlation was found between BMI and ultimate failure load of the CFL $(r=.919 ; P$ $=.01)$. A non-significant relationship was found between the mechanical properties and both age and weight. The ATFL avulsed from the fibula four times, the CFL avulsed from the fibula twice, the PTFL avulsed from the talus twice and all remaining failures were mid-substance.

\section{CONCLUSION}

The results identify the forces required to induce failure of the individual ligaments of the LCL complex and the related failure modes of individual ligaments. A correlation may exist between BMI and the ultimate failure load of the CFL and PTFL, although a greater sample size is required for confirmation.

Keywords

Characterisation; Ankle; Ligament; Sprain

\section{Introduction}

The lateral collateral ligament ( $L C L$ ) complex of the ankle (see Figure 1), consists of the anterior talofibular ligament (ATFL), calcaneofibular ligament (CFL) and posterior talofibular ligament (PTFL). The LCLs of the ankle are collectively responsible for the stabilisation of the talocrural joint on the lateral side and the CFL also plays a role in the stabilisation of the subtalar joint. 


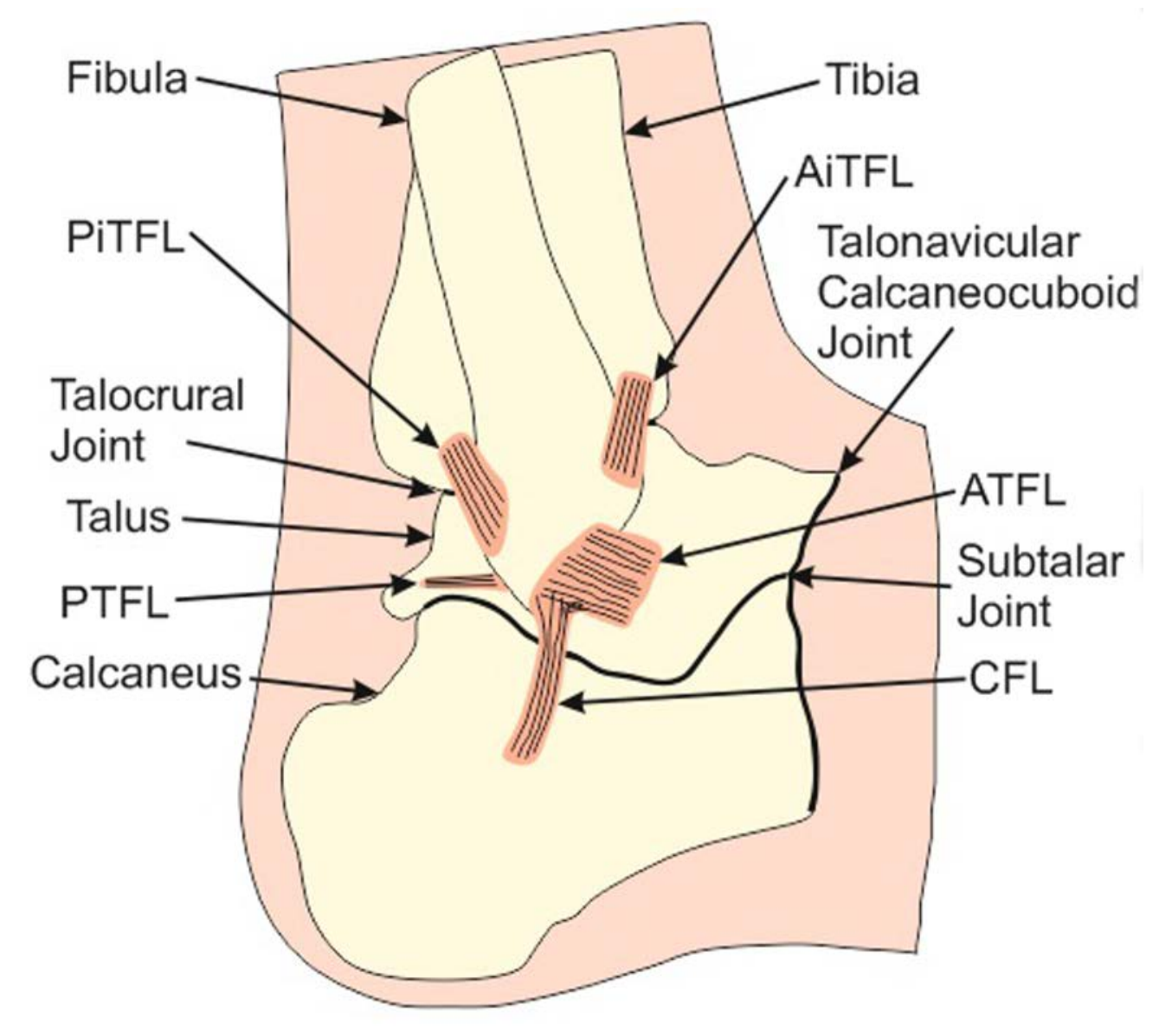

40

Figure 1. Lateral view of the ankle highlighting the LCL complex (ATFL, CFL \& PTFL), the syndesmosis (AiTFL \& PiTFL), the talocrural joint, the subtalar joint, the talonavicularcalcaneocuboid joint and the bones of the ankle(fibula, tibia, talus and calcaneus).

The ATFL is the most frequently injured LCL in a typical lateral ankle sprain, followed by the CFL and finally the PTFL. ${ }^{3,16}$ In cases of severe sprain or in people, such as elite athletes, wherein whom restoration of stability is important, surgical stabilisation may be performed. The current preferred standard is the Broström-Gould procedure in which ruptured ligaments are stabilised with sutures. If this approach is inadequate or has failed or if the patient has an increased BMI, general ligament laxity or is a high-demand athlete, then stabilisation with synthetic ligaments may be attempted. ${ }^{1}$

Natural ligaments exhibit a viscoelastic response to strain, starting with a progressively stiffer nonlinear toe region followed by a linear loading region. The response of ligamentous tissue is believed to be strain rate dependent due to the 
inherent viscoelastic nature of the tissue. ${ }^{6}$ This viscoelasticity causes ligaments to display hysteresis, due to the fluid component of the ligament being redistributed and balanced by the stress carried by the solid component of the ligament. When the lateral collateral ligament of the knee was tested at strain rates greater than 100 $\% . \mathrm{s}^{-1}$, a strain rate representative of inducing sprain in real-world events, it was found that the strain-rate dependency of the ligament can be neglected as there is insufficient time for appreciable ligament relaxation. ${ }^{6}$ The ligaments of the ankle have been reported to be generally insensitive to strain rate. ${ }^{9}$ Conversely, the mechanical properties of the LCLs have been reported to be significantly affected by strain rates both above and below $100 \% . \mathrm{s}^{-1} .^{3}$

Research articles detailing the mechanical characteristics of the LCL complex are scarce. ${ }^{3,9,16,17}$ None of the previous papers report mechanical characteristics of the LCL complex tested at realistic sprain-like strain rates. Attarian et al. (1985) and Funk et al. (2000) characterised the LCL complex at strain rates considerably higher than those which occur during a sprain event. ${ }^{3,9}$ Although ligaments are considered relatively insensitive to strain rates over $100 \% . \mathrm{s}^{-1}$ the effect on the failure mode of the ligaments is not understood. The absence of literature on this topic is potentially due to the difficulty faced when gripping ankle ligament tissue, as previously reported. ${ }^{16} \mathrm{~A}$ lack of published work in this area has hindered the understanding of the mechanical requirements and failure modes of synthetic interventions for lateral ankle sprain.

This study aimed to improve understanding of the mechanical properties and failure modes of the LCL complex at strain rates representative of real-world sprain events.

\section{Materials and Methods}

\subsection{Samples}


Six fresh frozen human cadaveric feet, sourced from MedCure (USA), were used in the study. Ethical approval was granted by the University of Leeds Research Ethics Committee (MEEC 15-020). Exclusion criteria for the tissues included a reported prior lower limb trauma or surgery, or a history of diabetes. The mean $( \pm 95 \%$ confidence intervals) donor age was $56.2 \pm 12.2$ years, BMl was $22.3 \pm 2.9 \mathrm{~kg} \cdot \mathrm{m}^{-2}$ (normal) and there were three males and three females. A summary of donor information is shown in Table 1.

Table 1. Tissue donor demographic details. The mean and $95 \%$ confidence interval $(\mathrm{Cl})$ is given for age, weight and body mass index (BMI). ( $\mathrm{M}$ - male, F - female, A.A - African American, C-Caucasian, $R-$ right \& $L-$ left).

\begin{tabular}{|c|c|c|c|c|c|c|}
\hline Sample & Age (years) & Sex & Race & Weight (kg) & BMI $\left(\mathrm{kg} \cdot \mathrm{m}^{-2}\right)$ & L/R Foot \\
\hline 1 & 72 & $M$ & A.A & 71 & 22.4 & $R$ \\
\hline 2 & 60 & $\mathrm{~F}$ & $\mathrm{C}$ & 53 & 18.9 & $\mathrm{~L}$ \\
\hline 3 & 49 & $F$ & $\mathrm{C}$ & 49 & 20.9 & $R$ \\
\hline 4 & 61 & $M$ & $\mathrm{C}$ & 66 & 21.4 & $R$ \\
\hline 5 & 38 & $M$ & $\mathrm{C}$ & 85 & 27.0 & $\mathrm{~L}$ \\
\hline 6 & 57 & $\mathrm{~F}$ & $\mathrm{C}$ & 61 & 23.2 & $\mathrm{R}$ \\
\hline Mean & 56.2 & - & - & 64.1 & 22.3 & - \\
\hline$\pm \mathrm{Cl}$ & \pm 12.2 & & & \pm 13.8 & \pm 2.9 & \\
\hline
\end{tabular}

\subsection{Sample Preparation}

The feet were stored in a $-80^{\circ} \mathrm{C}$ freezer, compliant with the Human Tissue Act, until they were tested. Samples were thawed for 48 hours at $4{ }^{\circ} \mathrm{C}$ in a refrigerator prior to dissection. After at least 24 hours of thawing, each foot was imaged, at a resolution of $82 \mu \mathrm{m}$, using a SCANCO Medical xtreme CT scanner (SCANCO Medical, Brüttisellen, Switzerland). Each scan lasted approximately 90 minutes and was performed to ensure no major undiagnosed damage was present.

The LCL complex was dissected intact from each foot while preserving the syndesmosis joint for future study, as shown in Figures $2 \& 3$. Firstly, all fascia and 
soft tissue were dissected from around the ankle by a foot and ankle specialist

101 consultant orthopaedic surgeon. Next, the forefoot was removed by transecting along the talonavicular calcaneocuboid joint. Using an oscillating bone saw, a sagittal cut was made through the entirety of the calcaneus and talus, as shown in Figure 2, Panel B. The lateral ankle complex was then removed by a transverse cut through the fibula, separating the LCL complex from the syndesmosis, as shown in Figure 2, Panel C. The cut was made from in-between the attachment points of the ATFL and anterior inferior talofibular ligament (AiTFL) to in-between the attachment points of the PTFL and posterior inferior talofibular ligament (PiTFL). The talus was then split in half with a coronal cut creating an anterior and posterior bone attachment segment for the ATFL and PTFL, respectively. Finally, the calcaneus was reduced in size and shaped to fit within the gripping fixture by performing two parallel coronal cuts either side of the attachment point and one transverse cut distally to the attachment point, as shown in Figure 3. The tissue hydration level of the ankle complex was maintained by wrapping the complex in phosphate-buffered saline (PBS) soaked paper towel. ${ }^{11}$ 


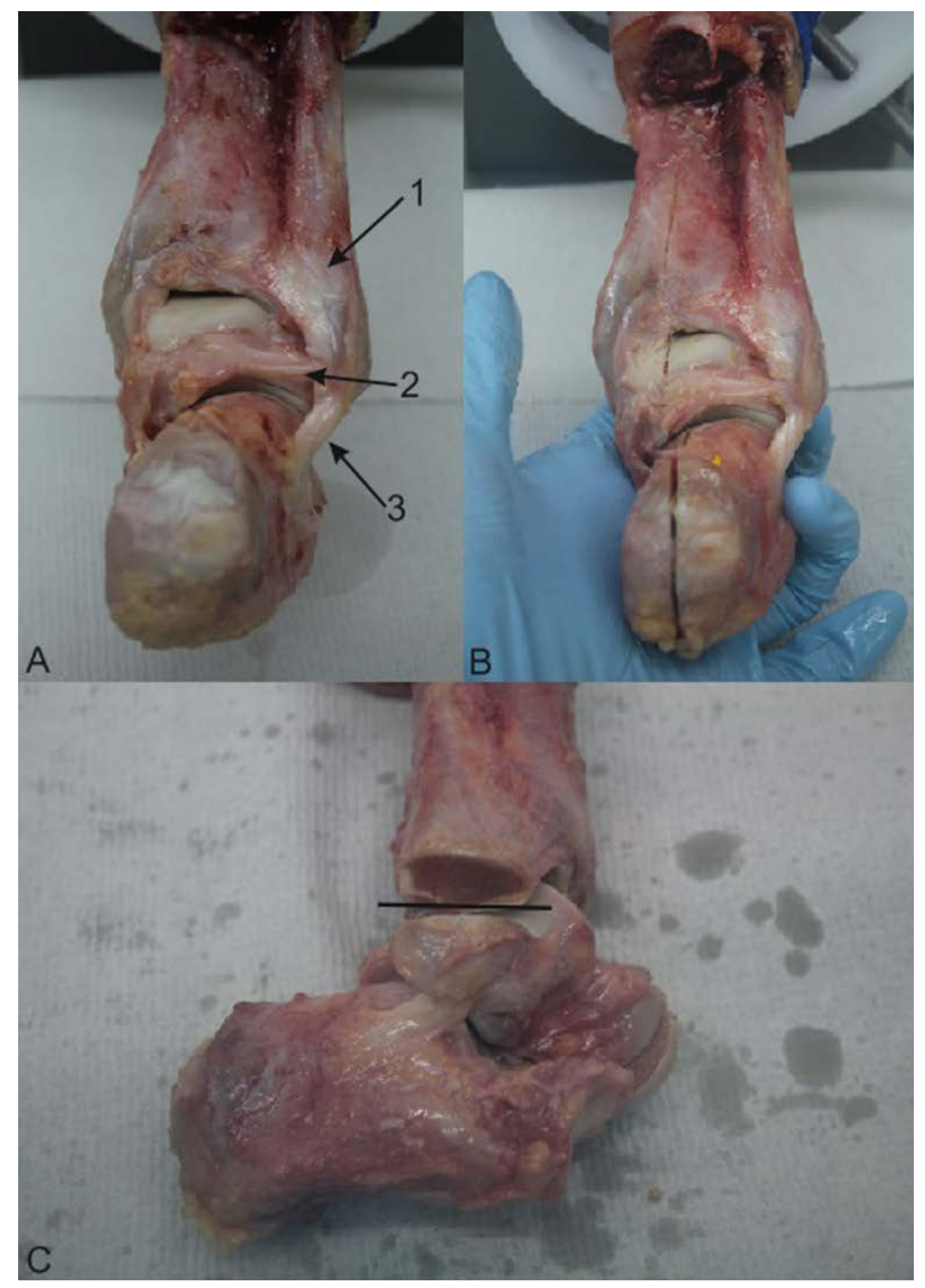

117 Figure 2. The dissection protocol employed to remove the $L C L$ complex from the rearfoot. A illustrates the intact rearfoot and provides a clear view of the PiTFL (1), PTFL (2) and CFL (3). B illustrates the sagittal cut made to separate the medial and lateral aspects of the rearfoot. C illustrates the transverse cut (black line) made through the fibula to separate the LCL complex and syndesmosis. 


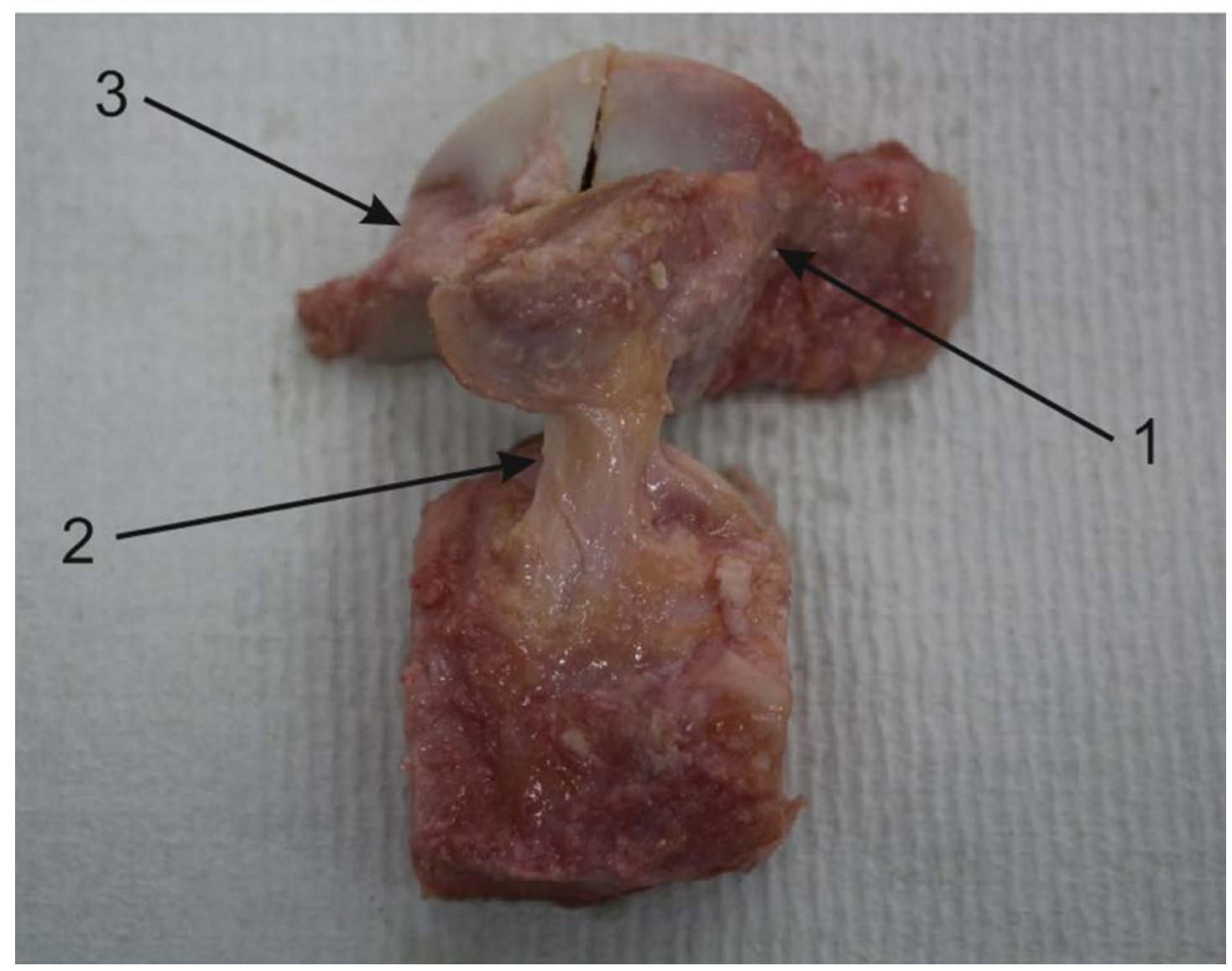

Figure 3. The LCL complex fully dissected prior to testing. The coronal cut into talus has been performed, creating separate bone pieces for the ATFL and PTFL, and the calcaneus has been shaped to fit within the bespoke testing grip. The ATFL (1), CFL (2) and PTFL (3) are shown.

Post-dissection ligament lengths were measured using Vernier callipers with the

ligaments orientated in line with their collagen fibres and the slack in the ligament was removed by hand. The ligaments were measured once, from the centre of one insertion to the centre of the other.

\subsection{Testing Protocol}

Each ligament of the LCL complex was tested individually whilst the complex was kept intact. The CFL was characterised first, then the ATFL followed by the PTFL.

Tissue rehydration was performed to ensure the viscoelastic nature of ligaments could act efficiently during the testing. Immediately before the characterisation of the CFL, the complex was submerged in PBS for 30 minutes. The complex was then submerged for 15 minutes prior to testing the ATFL and a further 15 minutes prior to 


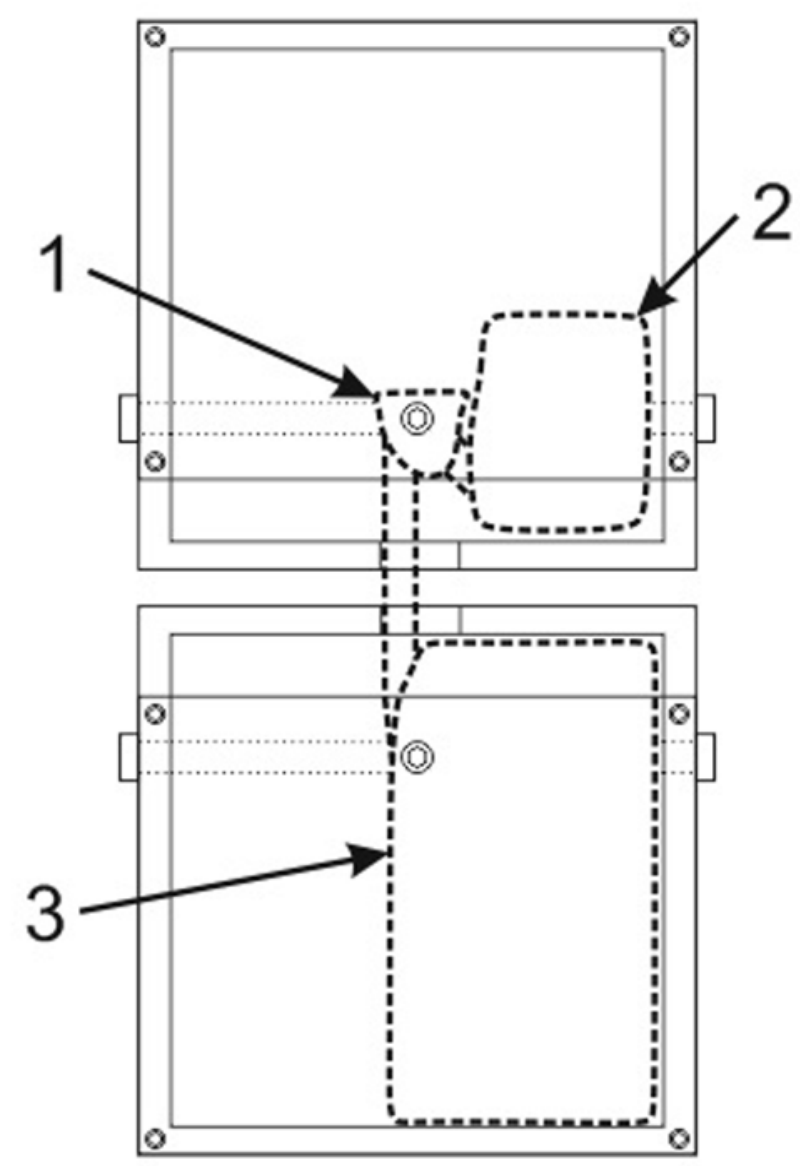

Figure 4. The LCL complex fixed into the bespoke gripping fixture with the CFL prepared for characterisation. The ATFL and PTFL, and their bony attachments from the fibula (1) to the talus (2) are within the top pot and the calcaneus (3) is within the bottom pot.

The mechanical characterisation was performed using an Instron ElectroPuls E10000, with a $1 \mathrm{kN}$ load cell (Instron, Buckinghamshire, UK). A floating joint was used to attach the top grip to the Instron to correct for any unintended malalignment within the setup. 
151

152

occurred within the specimens. ${ }^{14}$ Fifteen cycles of preconditioning following a sinusoidal waveform, ranging between two Newtons and a load value corresponding to $3.5 \%$ strain, were performed at a frequency of $0.83 \mathrm{~Hz}$. The $3.5 \%$ strain value represents the minimum amount of strain accumulated by any of the LCLs during one step of a normal walking cycle (10 degrees dorsiflexion through to 20 degrees plantarflexion). ${ }^{7}$ The preconditioning load values representing $3.5 \%$ strain were determined in a preliminary test of each ligament tested under strain control at a rate of $10 \% . \mathrm{s}^{-1}$. The frequency of $0.83 \mathrm{~Hz}$ is equivalent to the rate of normal walking (approximately one full gait cycle per second).

Following preconditioning, the specimens were then ramp loaded to failure at a strain rate of $100 \% . \mathrm{s}^{-1}$. A strain rate of $100 \% . \mathrm{s}^{-1}$ was selected to be representative of sprain, having previously been suggested to be a suitable injury strain rate for anterior cruciate ligament injury. ${ }^{4}$ The following equation, incorporating real-world inputs, also suggests that a strain rate of $100 \% . \mathrm{s}^{-1}$ is appropriate to replicate ankle ligament sprain.

Where $\dot{\varepsilon}$ is the strain rate, $\Delta L$ is the change in length of the ATFL from neutral position to maximum plantarflexion $(4.5 \mathrm{~mm}),{ }^{2} \mathrm{~L}$ is the length of the ATFL in the neutral position $(16.3 \mathrm{~mm})^{2}$ and $t$ is the time taken for the sprain motion of an ankle $(0.3 \mathrm{~s}) .^{8}$

\subsection{Data Analysis}

The mode of failure was determined via physical and visual examination of the specimens. Any specimens where the ligament had torn away from bone, torn cartilage away from bone or torn a small fragment of bone away from bone were 
substance failures. After the experimental testing, post-processing was completed to

177 calculate the ultimate failure load and stiffness of each ligament from each donor.

178 The linear stiffness value (k1) was calculated using a custom Matlab algorithm. ${ }^{11}$

179 Mean values and $95 \%$ confidence intervals for the ligament ultimate failure load,

180 stiffness and length, as well as the donor BMI, weight and age were calculated for

181 the ATFL, CFL and PTFL. A repeated measures ANOVA with a Greenhouse-Geisser

182 correction $(p<.01)$ was performed to calculate any significant differences in ultimate

183 failure load or stiffness between the ATFL, CFL and PTFL. Analysis of the data

184 stratified by age, weight and BMI was performed to identify any potential

185 correlations with these patient-specific factors and both ultimate failure load and

186 stiffness. Correlations were calculated for the ATFL, CFL and PTFL individually using a

187 two-tailed Pearson correlation test $(p<.01)$.

\section{Results}

The post-dissection ligament lengths used to calculate the ligament specific

190

191

192

193

194

195

196

197 preconditioning limits are provided in Table 2. The CFL was the longest of the three ligaments forming the LCL complex, with mean ( $\pm 95 \% \mathrm{Cl}$ ) length of $20.0 \pm 1.9 \mathrm{~mm}$. The PTFL and ATFL followed in order but were similar in length with mean lengths of $13.4 \pm 3.2 \mathrm{~mm}$ and $12.6 \pm 0.9 \mathrm{~mm}$, respectively.

Table 2. Ligament lengths $(\mathrm{mm})$ for each individual ligament and the mean ligament length and $95 \%$ confidence intervals (Cl) for ATFL, CFL and PTFL.

\begin{tabular}{cccc}
\hline Sample & ATFL & CFL & PTFL \\
\hline $\mathbf{1}$ & 11.62 & 17.60 & 10.50 \\
\hline
\end{tabular}




\begin{tabular}{cccc}
\hline $\mathbf{2}$ & 11.76 & 20.66 & 14.66 \\
\hline $\mathbf{3}$ & 12.90 & 19.24 & 10.54 \\
\hline $\mathbf{4}$ & 13.50 & 23.00 & 18.34 \\
\hline $\mathbf{5}$ & 12.08 & 20.06 & 14.80 \\
\hline $\mathbf{6}$ & 13.54 & 19.66 & 11.66 \\
\hline Mean $\pm \mathbf{C l}$ & $12.6 \pm 0.9$ & $20.0 \pm 1.9$ & $13.4 \pm 3.2$ \\
\hline
\end{tabular}

198

199 The mechanical characterisation results for the ATFL, CFL and PTFL are shown in

200

201

202

203

204

205

206

207

Table 3. The CFL had the highest mean ultimate failure load $( \pm 95 \% \mathrm{Cl})$ of $367.8 \pm$

79.8 N followed by the PTFL $351.4 \pm 110.8 \mathrm{~N}$, while the ATFL was the weakest $263.6 \pm$

164.3 N. No significant differences were found for the ultimate failure load $(p=.24)$

or stiffness $(p=.30)$ between the ATFL, CFL and PTFL.

Table 3. The mean and $95 \%$ confidence intervals $(\mathrm{Cl})$ for the ultimate failureload and stiffness results of the ATFL, CFL and PTFL. As well as the failure mode ( $\mathrm{A}-$ avulsion and $\mathrm{M}-$ mid-substance) and avulsion location.

\begin{tabular}{lccc}
\hline & ATFL & CFL & PTFL \\
\hline $\begin{array}{l}\text { Mean } \\
\text { Ultimate }\end{array}$ & $263.6 \pm$ & $367.8 \pm$ & $351.4 \pm$ \\
$\begin{array}{l}\text { Failure Load } \\
\pm \mathrm{Cl}(\mathrm{N})\end{array}$ & 164.3 & 79.8 & 110.8 \\
\hline $\begin{array}{l}\text { Mean } \\
\begin{array}{l}\text { Stiffness } \pm \\
\mathrm{Cl}(\mathrm{N} / \mathrm{mm})\end{array}\end{array}$ & $44.7 \pm$ & $45.8 \pm$ & $59.0 \pm$ \\
\hline $\begin{array}{l}\text { Failure } \\
\text { Mode } \\
\text { (A/M) }\end{array}$ & 46.6 & 19.0 & 10.7 \\
\hline $\begin{array}{l}\text { Avulsion } \\
\text { Site }\end{array}$ & Fibula & Fibula & Talus \\
\hline
\end{tabular}

The ratio of avulsions to mid-substance failures was similar for the ligament types tested, as detailed in Table 3. The ATFL avulsed from the fibula in four of the six tests, the CFL avulsed from the fibula in two of the six tests and the PTFL avulsed from the talus in two of the six tests. No systematic differences in ultimate failure load or stiffness were identified between the different failure modes. When avulsion 
did occur, the site of avulsion was consistent amongst ligament types (see Table 3).

Figures $5 \mathrm{~A}$ and $5 \mathrm{~B}$ illustrate clear examples of a mid-substance failure and avulsion, respectively.

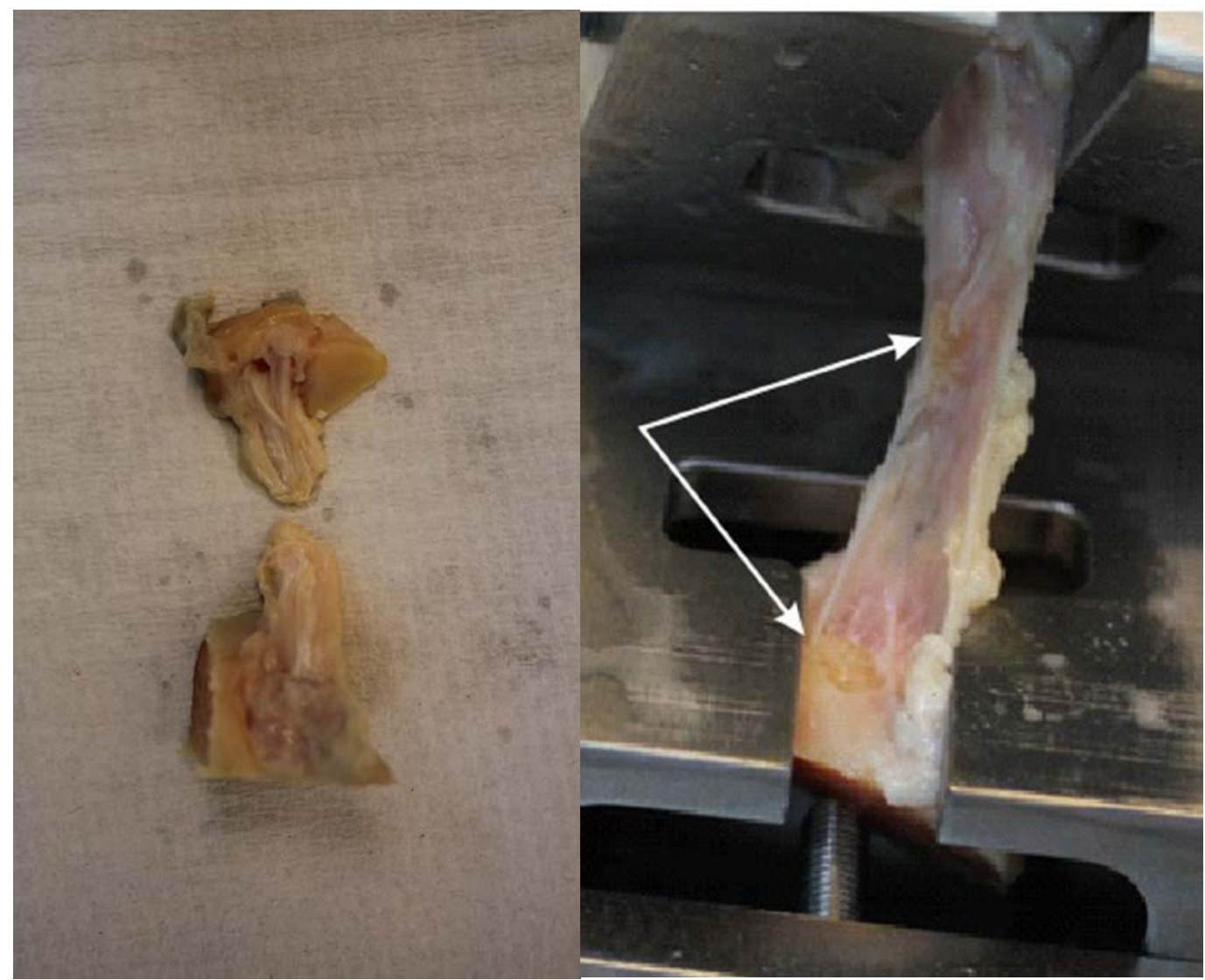

Figure 5. A) A mid-substance failure where intra-ligamentous failure has occurred. B) An avulsion failure where a fragment of bone has also been avulsed from the bone surface (white arrows).

The correlation results for the ultimate failure load and stiffness to the patientspecific factors: BMI, weight and age are presented in Table 4. The ultimate failure load of the CFL was found to have a significant strong positive Pearson correlation with BMI $(r=.92 ; p=.01)$. The ultimate failure load of the ATFL and PTFL had nonsignificant Pearson correlation scores $(r=.18 ; p=.73$ and $r=.65 ; p=.16$, respectively). A non-significant relationship was found for both age and weight with relation to both the ultimate failure load and stiffness of the ATFL, CFL and PTFL. Any relationship identified between BMI and stiffness of the ATFL $(r=-.05 ; p=.92)$, CFL ( $r$ $=.22 ; p=.68)$ and PTFL $(r=-.01 ; p=.98)$ was also negligible. 


\begin{tabular}{llccc}
\hline \multicolumn{2}{c}{ Ligament Property } & PSF & r-value & p-value \\
\hline Failure Load & ATFL & BMI & .184 & .727 \\
& CFL & BMI & $.919^{*}$ & .010 \\
& PTFL & BMI & .650 & .162 \\
\cline { 2 - 5 } & ATFL & Weight & .516 & .395 \\
& CFL & Weight & .874 & .023 \\
& PTFL & Weight & .327 & .527 \\
\cline { 2 - 5 } & ATFL & Age & .560 & .248 \\
& CFL & Age & -.273 & .600 \\
& PTFL & Age & -.496 & .317 \\
\hline Stiffness (k1) & ATFL & BMI & -.052 & .922 \\
& CFL & BMI & .216 & .681 \\
& PTFL & BMI & -.013 & .981 \\
\cline { 2 - 4 } & ATFL & Weight & .176 & .738 \\
& CFL & Weight & .410 & .419 \\
& PTFL & Weight & .000 & .999 \\
\cline { 2 - 4 } & ATFL & Age & .750 & .086 \\
& CFL & Age & -.397 & .436 \\
& PTFL & Age & -.340 & .510
\end{tabular}

231 The ultimate failure load results of the ATFL, CFL and PTFL are plotted against BMI in

232 Figure 6 with the results for all three ligaments of each donor aligned vertically

233 according to the donor's BMI. There is no evidence of a systematic tendency for the 234 ultimate failure load to vary by ligament type either within or between donors.

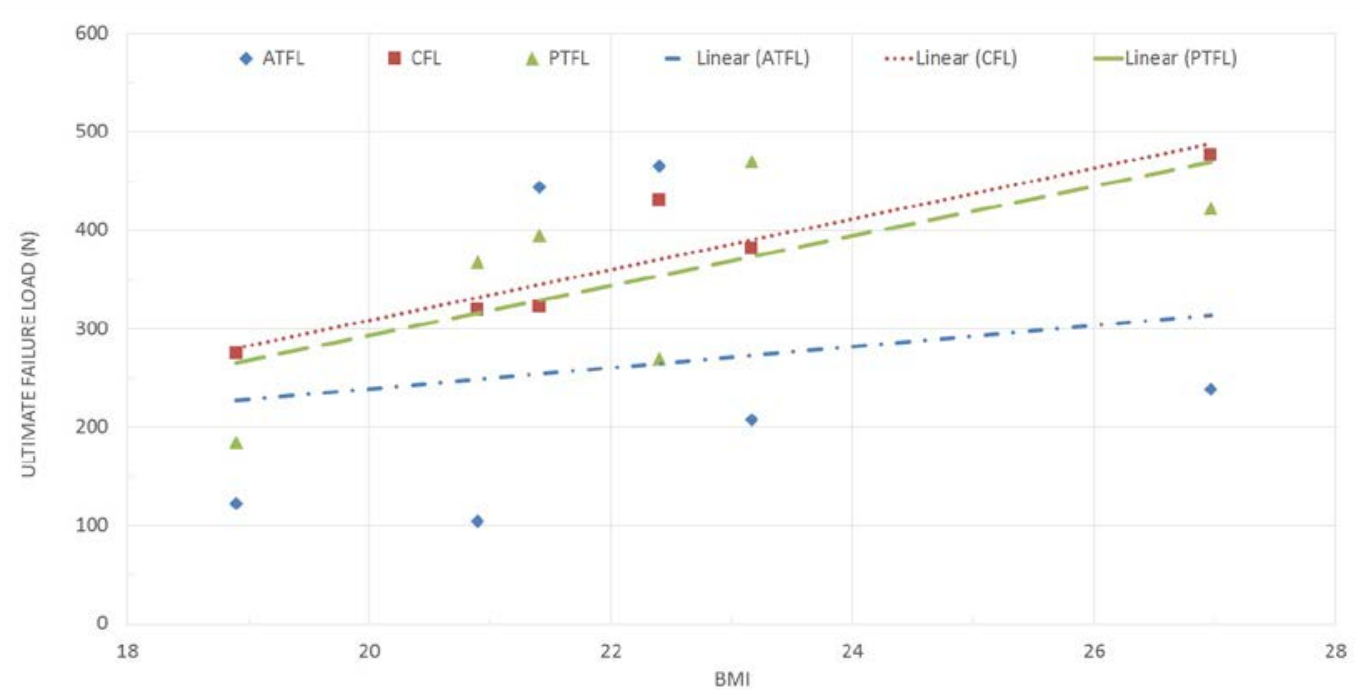

Figure 6. A graphical representation of the relationship between BMI and ultimate failureload. The three ligaments of each donor arevertically aligned according to the BMI of the donor. The ATFL is shown by blue diamond markers, the CFL by red square markers and the PTFL by green triangle 

dashed).

241

\section{Discussion}

The aim of this study was to improve understanding of the mechanical properties and failure modes of the LCL complex when strained at a rate representative of ankle sprain events in real-life. The mechanical characteristics of the entire LCL complex when loaded at a realistic sprain-like strain rate $\left(100 \% . \mathrm{s}^{-1}\right)$ are reported. The mean ultimate failure load results concur with previously published work, that the CFL and PTFL provide similar levels of support under load and that the ATFL is the weakest. ${ }^{3,16}$ There is however, a large amount variability between specimens, as shown in Figure 6, and there was no clear pattern for which ligament is the strongest or weakest at an individual donor level. Whilst St Pierre et al. (1983) only reports tensile strength of the ATFL they do so, in most cases, for each foot of each individual donor highlighting the substantial variability in ATFL failure load, ranging from $44 \mathrm{~N}$ to $556 \mathrm{~N}$. Notably, the ATFL, which is widely established as the weakest $\mathrm{LCL}$, was the strongest for two donors in this study, contradicting the general consensus. ${ }^{3,16}$ The widely established view that the ATFL is the weakest of the LCLS could therefore be incorrect for some people. The cause of this finding is likely multifactorial and a much larger sample size and in-depth patient information is required to substantiate any hypothesis.

Stiffness results in this study are similar to those previously reported by Attarian et al. (1985) who strained the LCLs at strain rates considerably higher than $100 \% . \mathrm{s}^{-1}$. This paper therefore supports the theory that the strain-rate dependency of ligaments can be neglected when tested at strain rates greater than $100 \% . s^{-1} .^{6,9}$ The current findings indicate a range of indicative ultimate failure loading requirements that can further inform the mechanical property specifications for synthetic ankle 
ligaments. Through improved matching of the mechanical properties, particularly the stiffness, of synthetic ligaments to their natural counterparts joint mobility and stability have the potential to also improve.

Both mid-substance failure and avulsion are abundantly prevalent as failure modes of the LCLs. Categorisation of the failure mode is somewhat subjective due to the fibrous nature of ligamentous failure, the difficulty faced differentiating between failure modes and the lack of a standardised definition of avulsion. The location of ligament avulsion was consistent, at the fibula for the ATFL and CFL and at the talus for the PTFL. Siegler et al. (1988) found the AFTL to avulse $58 \%$ of the time and the CFL and PTFL to avulse in $70 \%$ of tests, with remaining specimen failing midsubstance. ${ }^{16}$ Attarian et al. (1985) reported eight mid-substance failures and four talar avulsions for the ATFL, eight mid-substance failures, four calcaneal avulsions and four fibula avulsions for the CFL and four mid-substance failures for the PTFL. ${ }^{3}$ St Pierre et al. (1983) reported 18 talar avulsions, 16 mid-substance failures and two unknown failures. ${ }^{17}$

The location of ATFL avulsion in this study is inconsistent with those previously reported and an explanation as to why is unclear. Possible explanations include the status of the fibula, the orientation of the ligament or the vastly different strain rates. The fibula was intact for testing in the studies by St Pierre et al. (1983) and Attarian et al. (1985) whereas in this study the fibula was split reducing the amount of bone to be gripped. The orientation of the specimen may differ slightly between this study and the two studies highlighted due to the fibula not being intact, although all studies attempted tensile testing with fibre alignment. The prevalence of avulsion and mid-substance failures are however comparable. The high prevalence of avulsions could be due to the significantly higher local strain proximal to the attachment site of ligaments compared to the central region. ${ }^{18}$ The failure 
mechanism of a ligament is an important consideration prior to a ligament repair being performed as the fixation method may differ depending on whether the ligament needs reattaching to bone or to ligament.

A potentially noteworthy finding was the positive correlation between $\mathrm{BMI}$ and ultimate failure load values for the ligaments of the LCL complex, specifically the CFL. This finding, from a sample size of six, suggests that the CFL of individuals with a higher BMI have a greater load bearing capacity than those with a lower BMI. This is most likely due to the adaptive remodelling nature of ligamentous structures, as individuals with a greater BMI are likely to apply more stress to the ligament, increasing strength over time..$^{5}$ The $\mathrm{BMI}$ of an individual could therefore be an important factor when selecting the appropriate material properties of a synthetic intervention, and notably people with a high BMI who are more often candidates for a synthetic ligament replacement. ${ }^{1}$ Therefore, the load bearing capacity of the synthetic, and their fixation devices, should match the mean ultimate failure load to ensure the synthetic does not subsequently fail. The stiffness of the synthetic material, along with the tension applied upon insertion, is arguably more important. A stiffness that is too high could reduce the joint mobility and too low could affect the stability of the joint. Therefore it could be recommended that the stiffness of the synthetic material is also matched to that of the natural tissue results reported.

The anatomy of ligaments is often depicted incorrectly in illustrations because of stylistic licence. The previously published pictorial essay does however provide detailed images of the ankle ligament anatomy. ${ }^{10}$ Figure 3 shows the attachment points of the ATFL and CFL to the fibula. These attachments are often illustrated as separate insertion points however as shown in Figure 3, the two ligaments commonly attach at the same insertion point on the fibula. It is suggested that the inferior aspect of the ATFL and CFL are connected by arciform fibres, ${ }^{15}$ thus forming 
the lateral fibulotalocalcaneal complex. ${ }^{12}$ This observation was also made when performing the dissections for this study. The results of this study however suggest that the connecting fibres are not of a sufficient strength to cause both the ATFL and CFL to rupture simultaneously. The CFL was tested first in every instance and the results of the ATFL are still similar to those previously published, where they were tested without the arciform fibres present. ${ }^{16}$

The limitations to the study predominantly centre on the use of human cadaveric tissue. The main limitation is the small sample size $(n=6)$. Research using donor cadaveric tissue should be minimised to only what is essential and performed with maximum efficiency and integrity out of respect for the donors. The characterisation of cadaveric human tissue may not reflect the same response as living tissue. However, ligamentous tissue primarily attributes its strength properties to the collagen fibres which form the majority of ligament structure. The collagen would not be greatly affected by the tissue being living or dead, providing it remains well hydrated and is stored appropriately to abate tissue degradation. Although the exclusion criteria required donors to have not reported any lower limb trauma we cannot be certain that a prior sprain had not occurred at some point during the donor's lifespan. It is estimated that ankle injury rates are approximately five and a half times higher than those registered in emergency departments. ${ }^{13}$ This could provide some explanation for the inconsistencies in strength between ligament types (Figure 6). Large variations in the results following the mechanical characterisation of ankle ligaments are also reported elsewhere. ${ }^{16}$ The use of elderly donor tissue to investigate sprain has previously been suggested to be a limitation of cadaver studies. An effort was therefore made when selecting donor specimens to obtain the youngest specimens possible (mean 56.2 years). A previous study however, reported no correlation between ultimate failure load and age for donors aged 17 to 54 when 
testing human anterior cruciate ligaments. ${ }^{4}$ The link identified between $\mathrm{BMI}$ and

344 ultimate failure load of the CFL and PTFL is based on a narrow range of BMI with only

345 one donor having a BMI outside of the normal range and the trend may not be

346 reflected in a population at the extremities of the BMI scale.

\section{Conclusion}

348 Limitations aside, the conditions of this study were carefully defined to reflect those experienced by individuals who would suffer an ankle sprain allowing for the entire LCL complex to be characterised at realistic sprain inducing strain rates. In the current study the ultimate failure load and stiffness of the ATFL, CFL and PTFL did not differ systematically but there was a tendency toward greater strength in people with a higher BMI. The maximum likely exposure loads, the BMI of the patient and the failure mode of the LCLs all appear to be factors to be further considered when selecting the material, repair or reconstruction technique to be used for surgical stabilisation of the sprained ankle.

\section{Acknowledgements}

The authors would like to acknowledge Dr Nagitha Wijayathunga for his contributions to the CT scanning of the samples. 


\section{References}

1. Ajis A, Younger ASE, Maffulli N. Anatomic Repair for Chronic Lateral Ankle Instability. Foot Ankle Clin. 2006;11(3):539-545. doi:10.1016/j.fcl.2006.07.005

2. de Asla RJ, Kozanek M, Wan L, Rubash HE, Li G. Function of anterior talofibular and calcaneofibular ligaments during in-vivo motion of the ankle joint complex. J Orthop Surg Res. 2009;4(1):7. doi:10.1186/1749-799X-4-7

3. Attarian DE, McCrackin HJ, DeVito DP, McElhaney JH, Garrett WE. Biomechanical characteristics of human ankle ligaments. Foot Ankle. 1985;6(2):54-58. doi:10.1177/107110078500600202

4. Blevins FT, Hecker AT, Bigler GT, Boland AL, Hayes WC. The Effects of Donor Age and Strain Rate on the Biomechanical Properties of Bone-Patellar Tendon-Bone Allografts. Am J Sports Med. 1994;22(3):328-333. doi:10.1177/036354659402200306

5. Bonnel F, ToullecE, Mabit C, Tourné Y. Chronic ankle instability: Biomechanics and pathomechanics of ligaments injury and associated lesions. Orthop Traumato/Surg Res. 2010;96(4):424-432. doi:10.1016/j.otsr.2010.04.003

6. BonnerTJ, Newell N, Karunaratne A, et al. Strain-rate sensitivity of the lateral collateral ligament of the knee. J Mech Behav Biomed Mater. 2015;41:261-270. doi:10.1016/j.jmbbm.2014.07.004

7. Colville MR, Marder RA, Boyle JJ, Zarins B. Strain measurement in lateral ankle ligaments. Am J Sports Med. 1990;18(2):196-200. doi:10.1177/036354659001800214

8. Fong DT-P, Hong Y, Shima Y, Krosshaug T, Yung PS-H, Chan K-M. Biomechanics of supination ankle sprain: a case report of an accidental injury event in the laboratory. Am J Sports Med. 2009;37(4):822-827. doi:10.1177/0363546508328102

9. Funk JR, Hall GW, Crandall JR, Pilkey WD. Linear and Quasi-Linear Viscoelastic Characterization of Ankle Ligaments.J Biomech Eng. 2000;122(1):15. doi:10.1115/1.429623

10. Golanó P, Vega J, de Leeuw PAJ, et al. Anatomy of the ankle ligaments: a pictorial essay. Knee Surgery, Sport TraumatolArthrosc. 2016;24(4):944-956. doi:10.1007/s00167-016-4059-4

11. Herbert A, Brown C, Rooney P, Kearney J, Ingham E, Fisher J. Bi-linear mechanical property determination of acellular human patellar tendon grafts for use in anterior cruciate ligament replacement. J Biomech. 2016;49(9):1607-1612. doi:10.1016/j.jbiomech.2016.03.041

12. Hertel J. Functional anatomy, pathomechanics, and pathophysiology of lateral ankle instability. J Ath/Train. 2002;37(4):364-375. doi:10.1017/CBO9781107415324.004

13. KemlerE, van de Port I, Valkenberg H, Hoes AW, Backx FJG. Ankle injuries in the Netherlands: Trends over 10-25 years. Scand J Med Sci Sports. 2015;25(3):331-337. doi:10.1111/sms.12248

14. Quinn KP, Winkelstein BA. Preconditioning is correlated with altered collagen fiber alignment in ligament. J Biomech Eng. 2011;133(6):575-579. doi:10.1115/1.4004205

15. Sarrafian SK. Anatomy of the Foot and Ankle. JBLippincott Co. 1983.

16. Siegler S, Schneck CD. The Mechanical Characteristics of the Collateral Ligaments of the Human Ankle Joint. Foot Ankle Int. 1988;8(5):234-242. doi:10.1177/107110078800800502

17. St Pierre RK, Rosen J, Whitesides TE, Szczukowski M, Fleming LL, Hutton WC. The tensile strength of the anterior tal ofibular ligament. Foot Ankle. 1983;4(2):83-85. 
18. Stouffer DC, Butler DL, Hosny D. The relationship between crimp pattern and mechanical response of human patellar tendon-bone units. J Biomech Eng.

409 1985;107(2):158-165. doi:10.1115/1.3138536

410 